\title{
Donepezil for Compulsive Behavior in Degenerative Dementia: Case Series
}

\author{
Biswa Ranjan Mishra ${ }^{1}$, Vanteemar S. Sreeraj ${ }^{2}$, Saranya Dhanashekaran ${ }^{2}$, Rituparna Maiti ${ }^{3}$ \\ Departments of ${ }^{1}$ Psychiatry and ${ }^{3}$ Pharmacology, All India Institute of medical Sciences, Bhuvaneshwar, ${ }^{2}$ Department of Psychiatry, National \\ Institute of Mental Health and Neurosciences, Bangalore, India
}

\begin{abstract}
Diagnosing and managing dementia, presenting with compulsions is challenging. Presented are three cases, a possible representative subset of the Donepezil responders. Selective degeneration of dorsolateral prefrontal cortex networking striatum leading to compulsions would be amenable to cholinergic modulation.
\end{abstract}

KEY WORDS: Repetition compulisons; Psychosis; Dementia; Major neurocognitive disorder; Donepezil.

\section{INTRODUCTION}

The onset of repetitive, compulsive behaviors at late age usually stems from an organic basis or can be the presentation of a progressive dementing process. ${ }^{1,2)}$ Frontotemporal dementia (FTD) is recognized as the commonest neurological cause for new-onset compulsive behaviors in middle to late life. ${ }^{1)}$ But it can be a presenting symptom in Alzheimer's and other dementia. ${ }^{3,4)}$ In relation to the treatment of the behavioral symptoms in dementia there are limited studies, and anticholinesterase inhibitors such as donepezil have shown mixed results, with worsening of behaviors reported by few studies. ${ }^{5,6)}$ Here we present a series of three cases of degenerative dementia with an onset characterized by compulsive and psychotic behavior, which heralded the onset of a dementing process with a seemingly good response to donepezil including a dramatic reduction of the behavioral symptoms.

Received: October 24, 2016/Revised: January 19, 2017

Accepted: January 20, 2017

Address for correspondence: Vanteemar S. Sreeraj, DPM, DNB (Psychiatry)

Department of Psychiatry, National Institute of Mental Health and Neurosciences, Bangalore 560029, India

Tel: +91-9902597697, Fax: +91-80-26562121/26564830

E-mail: vs8sreeraj@yahoo.com

ORCID: https://orcid.org/0000-0002-2946-1228

\section{CASE}

\section{Case 1}

A 64 years old hypertensive female presented with an acute onset 6 months' history of forcing her grandson to repeatedly wash the toilet without citing any reasons, compulsive checking of her belongings, intermittent irritability, muttering to herself and reduced sleep with poor attention and insight. Psychotic symptoms and cognitive performance worsened during clomipramine trial of 150 $\mathrm{mg} /$ day. A possibility of late onset psychotic disorder was considered. Addition of tablet risperidone $3 \mathrm{mg} /$ day reduced irritability, hallucinatory behavior but not compulsions, with the appearance of word finding, naming difficulties and forgetfulness impacting daily activities. Computed tomography (CT) scan of brain and detailed neuropsychological assessment revealed a degenerative process (Table 1). Addition of oral donepezil $10 \mathrm{mg}$ /day to ongoing risperidone brought slight improvement in the domains of ideational fluency and vigilance and a significant reduction in repetitive behavior.

\section{Case 2}

A 75 years old male presented with 8 months' history of insidious onset of illness, characterized by "sand" or "crawling of insects" like tactile sensations under the eyelids, resulting in compulsive cleaning and washing of eyelids and body parts, along with fleeting 2nd person audi-

(ㄷ) This is an Open-Access article distributed under the terms of the Creative Commons Attribution Non-Commercial License (http://creativecommons.org/licenses/by-nc/4.0) which permits unrestricted non-commercial use, distribution, and reproduction in any medium, provided the original work is properly cited. 
Table 1. Neurocognitive domains and structural involvement in the presented cases

\begin{tabular}{lccc}
\hline \multicolumn{1}{c}{ Neuro-cognitive domains involved } & Case 1 & Case 2 & Case 3 \\
\hline Attention, concentration \& vigilance & $*$ & & $*$ \\
Working memory & $*$ & $*$ & $*$ \\
New learning ability (verbal \& visual) & $*$ & $*$ & $*$ \\
Delayed recall & & $*$ & $*$ \\
Motor execution & $*$ & $*$ & Bilateral basi-temporal \& \\
Ideational fluency & $*$ & frontal (left $>$ right)) \\
Verbal fluency & Bilateral basi-temporal \& & frontal \\
Comprehension & &
\end{tabular}

$\mathrm{CT}$, computed tomography.

*Cognitive domains impaired.

tory hallucinations, irritability and reduced sleep. A provisional diagnosis of monosymptomatic hypochondriacal psychosis, with differential diagnosis of obsessive compulsive disorder (OCD) with poor insight/organic psychotic disorder was considered. On tablet risperidone 3 $\mathrm{mg} /$ day, the behavioral symptoms other than hallucinations worsened. The CT scan of brain and neuropsychological tests were suggestive of major neurocognitive disorder (Table 1), tablet donepezil $10 \mathrm{mg} /$ day was gradually added to risperidone $2 \mathrm{mg} /$ day. On follow up after 4 months, there was a significant reduction in cleaning behavior without further deterioration in cognitive functions.

\section{Case 3}

A 73 years old hypertensive female came with 3 weeks' history of acute onset of repeatedly checking the doors, irritability, persecutory delusions, intermittent 3rd person auditory hallucination, impaired concentration and judgment. A provisional diagnosis of acute and transient psychotic disorder was kept, with a possibility of dementia in evolution. Patient showed significant reduction in the various dimension of delusions and hallucinations with risperidone $3 \mathrm{mg} /$ day. In view of the emerging neurocognitive disorder, which became apparent on neuropsychological assessment and imaging (Table 1), tablet donepezil $5 \mathrm{mg} /$ day was started. On next visit at $2 \mathrm{nd}$ month, interestingly there was significant reduction in the frequency of the repetitive behaviors, with improvement of performance in working memory and vigilance.

\section{DISCUSSION}

The compulsive behaviors in dementia are usually atypical, with lack of insight into the irrationality and excessive nature of the repetitive acts. ${ }^{1)}$ In our cases, the thought behind the repetitive behavior could hardly be elicited; however, appeared secondary to hypochondriacal or persecutory doubts. Furthermore, the additional rapid cognitive decline and deterioration in personality, were suggestive of a dementing process with repetitive behaviors being the initial symptom presentation. However, there were quite a few atypicalities in our cases, in relation to the nature and age of onset of the illness.

FTD which is the commonest degenerative etiology of compulsions usually emerges before 65 years of age with an insidious onset. Two of our three patients had an onset after 65 years. The perceptual disturbances at presentation, particularly with well-formed third person auditory hallucinations are another atypicality found in our cases. ${ }^{7)}$ Though rare, but schizophrenia like psychotic illness have been described in few histologically proven FTD cases, having relatively younger onset, with comorbid motor neuron disorder. ${ }^{8)}$

The association between late-onset compulsive symptoms and dementia may arise from at least 3 different scenarios. First, dementia may be the final end-point of some form of primary, resistant OCD. ${ }^{9)}$ Second, late-onset compulsive behaviors, or stereotypies and dementia may result from a common pathophysiologic basis, such as in FTD. ${ }^{10)}$ Finally, the association may result from the interaction between vulnerability towards obsessive compulsive (OC) symptoms and the nonspecific effects of a neu- 
rodegenerative process. ${ }^{3)}$ The combination of psychosis, compulsions and dementia found in our cases, can be explained by a common glutaminergic system dysfunction. Unstable prefrontal glutamatergic system with hyperglutamatergic orbitofrontal (OFC) and hypoglutamatergic dorsolateral prefrontal (DLPFC) regions has been theorized to be the pathophysiology behind the schizo-obsessive disorder. ${ }^{11)}$ Similar glutamate mediated degenerative process involving the DLPFC along with striatal circuits would be resulting in the symptom presentation in our cases.

Another variation was that, apart from repetitive behavior, all of them lacked the other classical symptoms of behavioral variant (bv) FTD as follow; apathy, disinhibition, asociality, and change in dietary choices. This lack of common 'frontal' bv FTD features such as disinhibition or apathy suggests relative sparing of OFC and ventromedial prefrontal cortices (VMPFC). ${ }^{12)}$ Language, memory and executive functions deficits implicates the temporal and DLPFC involvement. Moreover in stereotypic form of FTD, marked striatal changes and variable cortical involvement are reported, with advanced temporal rather than frontal lobe pathology. ${ }^{13)}$ Thus, the imaging, neuropsychological tests and symptoms in present series implicate prominent temporal region and DLPFC with relative sparing of OFC and VMPFC. The current cases though has features of selective frontal and temporal lobe involvement, they would not fit into the classical descriptions of any defined categories of dementia. Histopathological and genetic tests could have confirmed the diagnosis, which were not done due to the resource constraints.

The most important finding in our case series was the dramatic reduction in repetitive and compulsive behaviors with donepezil. Striatal circuits have dense array of cholinergic interneurons which have modulatory role in the repetitive behaviors and stereotypies. ${ }^{14)}$ Cholinergic depletion reportedly produces repetitive behaviors in animal models which responds to donepezil. ${ }^{15)}$ Donepezil is known to improve behavioral symptoms in Alzheimer's dementia. ${ }^{16)}$

Summary of the possible biological underpinnings is as follows: degeneration of cortical neurons at DLPFC and temporal regions might have resulted in hypoglutamatergic DLPFC and relative hyperglutamatergic OFC regions, the circuitry changes often seen in patients with schizo-obsessive disorder. Cortical glutamatergic neurons modulate the striatal output through GABA modulating cholinergic interneurons. ${ }^{14,17)} \mathrm{A}$ deficiency in cortical glutamate led to reduced striatal cholinergic activity, which in turn increased the OC symptoms. Donepezil by reducing the hydrolysis increases the concentration of acetylcholine in striatal synapses, thereby effectively modulating the excitatory-inhibitory balancing and ameliorates the OC symptoms. Kerchner et al. ${ }^{6}$ has suggested the possible efficacy of donepezil in a subset of patients with FTD. All three of our cases showed remarkable reduction in the repetitive behaviors after adding donepezil. We suggest that the patients with late age of onset, having an acute onset of compulsive and psychotic symptoms and with predominantly DLPFC/temporal lobe involvement could be a subset which responds to choline esterase inhibitors.

In conclusion, detailed neurocognitive and neuroimaging investigations are imperative to unmask dementias that present with complex atypical psychiatric manifestations. Role of donepezil in management of repetitive behaviors during degenerative process needs further systematic evaluation.

\section{REFERENCES}

1. Mendez MF, Perryman KM, Miller BL, Swartz JR, Cummings JL. Compulsive behaviors as presenting symptoms of frontotemporal dementia. J Geriatr Psychiatry Neurol 1997;10:154157.

2. Weiss AP, Jenike MA. Late-onset obsessive-compulsive disorder: a case series. J Neuropsychiatry Clin Neurosci 2000;12: 265-268.

3. Dondu A, Sevincoka L, Akyol A, Tataroglu C. Is obsessivecompulsive symptomatology a risk factor for Alzheimer-type dementia? Psychiatry Res 2015;225:381-386.

4. Nyatsanza S, Shetty T, Gregory C, Lough S, Dawson K, Hodges JR. A study of stereotypic behaviours in Alzheimer's disease and frontal and temporal variant frontotemporal dementia. J Neurol Neurosurg Psychiatry 2003;74:1398-1402.

5. Mendez MF, Shapira JS, McMurtray A, Licht E. Preliminary findings: behavioral worsening on donepezil in patients with frontotemporal dementia. Am / Geriatr Psychiatry 2007;15: 84-87.

6. Kerchner GA, Tartaglia MC, Boxer A. Abhorring the vacuum: use of Alzheimer's disease medications in frontotemporal dementia. Expert Rev Neurother 2011;11:709-717.

7. Iwahashi M, Netsu Y, Imamura T. [Auditory hallucination as an initial sign of frontotemporal dementia]. Brain Nerve 2012;64:697-702. Japanese.

8. Velakoulis D, Walterfang M, Mocellin R, Pantelis C, McLean C. 
Frontotemporal dementia presenting as schizophrenia-like psychosis in young people: clinicopathological series and review of cases. Br J Psychiatry 2009;194:298-305.

9. Nakaaki S, Murata Y, Shinagawa Y, Hongo J, Furukawa TA, Sato J, et al. A case of late-onset obsessive compulsive disorder developing frontotemporal lobar degeneration. J Neuropsychiatry Clin Neurosci 2007;19:487-488.

10. Ames D, Cummings JL, Wirshing WC, Quinn B, Mahler M. Repetitive and compulsive behavior in frontal lobe degenerations. J Neuropsychiatry Clin Neurosci 1994;6:100-113.

11. Venkatasubramanian G, Rao NP, Behere RV. Neuroanatomical, neurochemical, and neurodevelopmental basis of obsessive-compulsive symptoms in schizophrenia. Indian I Psychol Med 2009;31:3-10.

12. Schroeter ML, Raczka K, Neumann J, Yves von Cramon D. Towards a nosology for frontotemporal lobar degenerations-a meta-analysis involving 267 subjects. Neuroimage 2007;36: 497-510.
13. Snowden JS, Neary D, Mann DM. Frontotemporal dementia. Br J Psychiatry 2002;180:140-143.

14. Lim SA, Kang UJ, McGehee DS. Striatal cholinergic interneuron regulation and circuit effects. Front Synaptic Neurosci 2014;6:22.

15. Cutuli D, Foti F, Mandolesi L, De Bartolo P, Gelfo F, Federico $\mathrm{F}$, et al. Cognitive performances of cholinergically depleted rats following chronic donepezil administration. J Alzheimers Dis 2009;17:161-176.

16. Hansen RA, Gartlehner G, Webb AP, Morgan LC, Moore CG, Jonas DE. Efficacy and safety of donepezil, galantamine, and rivastigmine for the treatment of Alzheimer's disease: a systematic review and meta-analysis. Clin Interv Aging 2008;3:211225.

17. Doig NM, Magill PJ, Apicella P, Bolam JP, Sharott A. Cortical and thalamic excitation mediate the multiphasic responses of striatal cholinergic interneurons to motivationally salient stimuli. J Neurosci 2014;34:3101-3117. 